\title{
Dynamic Interactive Social Cognition Training in Virtual Reality (DiSCoVR) for social cognition and social functioning in people with a psychotic disorder: study protocol for a multicenter randomized controlled trial
}

Saskia A. Nijman ${ }^{1,2,8^{*}}$ D, Wim Veling ${ }^{2}$, Kirstin Greaves-Lord ${ }^{3,4,5}$, Rowina R. Vermeer ${ }^{6}$, Maarten Vos ${ }^{2}$, Catharina E. R. Zandee ${ }^{6}$, Daniëlle C. Zandstra ${ }^{7}$, Chris N. W. Geraets ${ }^{2}$ and Gerdina H. M. Pijnenborg ${ }^{1,8}$

\begin{abstract}
Background: Problems in social functioning (e.g., unemployment, social isolation), are common in people with a psychotic disorder. Social cognition is a treatment target to improve social functioning, as it is a proximal predictor of social functioning. Social Cognition Training (SCT) improves social cognition, but may not generalize (enduringly) to social functioning, perhaps due to insufficient opportunity to practice in daily-life social situations. Using virtual reality (VR) for SCT could address this problem, as VR is customizable, accessible, and interactive. We will test the effect of a VR SCT, 'DiSCoVR', on social cognition and social functioning in a randomized controlled trial (RCT).

Methods: In total 100 people with a psychotic disorder and deficits in social cognition will be recruited for this multicenter randomized controlled trial (RCT). Participants will be randomized to VR SCT (DiSCoVR) or VR relaxation training (VRelax; active control). DiSCoVR is a 16-session individual SCT, consisting of three modules: 1) emotion perception (recognizing facial emotions in a virtual shopping street); 2) social perception and theory of mind (observing social interactions between virtual characters and assessing their behavior, emotions and thoughts); and 3) application of higher-order social cognition in social interaction (role-playing personalized situations in VR). People receiving VRelax complete sixteen individual sessions, in which they receive psycho-education about stress, identify personal stressors, learn relaxation techniques, and explore relaxing immersive virtual environments. Assessments will be performed at baseline, post-treatment, and 3-month follow-up. Primary outcomes are emotion perception (Ekman 60 Faces), social perception and theory of mind (The Awareness of Social Inference Test). Secondary outcomes include social functioning (Personal and Social Performance Scale), experiences and social interactions in daily life (experience sampling of emotions, social participation and subjective experience of social situations), psychiatric symptoms (e.g., depression, perceived stress, anxiety, positive and negative symptoms) and (Continued on next page)
\end{abstract}

\footnotetext{
* Correspondence: s.a.nijman@umcg.nl

'Department of Psychotic Disorders, GGZ Drenthe, Dennenweg 9, PO Box 30007, 9404, LA, Assen, the Netherlands

${ }^{2}$ University Center of Psychiatry, University Medical Center Groningen,

University of Groningen, Hanzeplein 1, PO Box 30.001, 9700, RB, Groningen,

the Netherlands

Full list of author information is available at the end of the article
}

(c) The Author(s). 2019 Open Access This article is distributed under the terms of the Creative Commons Attribution 4.0 International License (http://creativecommons.org/licenses/by/4.0/), which permits unrestricted use, distribution, and reproduction in any medium, provided you give appropriate credit to the original author(s) and the source, provide a link to the Creative Commons license, and indicate if changes were made. The Creative Commons Public Domain Dedication waiver (http://creativecommons.org/publicdomain/zero/1.0/) applies to the data made available in this article, unless otherwise stated. 
(Continued from previous page)

self-esteem.

Discussion: To our knowledge, this will be the first RCT testing the efficacy of VR SCT. It will also investigate generalization to daily life social situations, the durability of treatment effects, and moderators and mediators of treatment success.

Trial registration: On December 5, 2017, this trial was registered prospectively in the Dutch Trial Register as NTR6863.

Keywords: Social cognition training, Virtual reality, Emotion perception, Theory of mind, Psychotic disorder, Social functioning

\section{Background}

For many people who have a psychotic disorder, it is a challenge to have meaningful daily activities, such as work, and to develop and maintain personal and romantic relationships; many of them experience problems in social functioning [1]. To function in daily life, it is necessary to adequately handle social situations and to be able to interpret the emotions, thoughts, intentions and behaviors of other people. These capabilities are generally referred to as social cognition. However, in people with a psychotic disorder, deficits in social cognition are common; a meta-analysis found large deficits in emotion perception and processing, social perception and theory of mind, compared to healthy controls [2]. Deficits in these key domains have been found to be important, proximal predictors for social functioning [3]. Social cognition has therefore become a target for psychosocial interventions aiming to improve social functioning through social cognitive training [4], a class of interventions also known as social cognition training (SCT).

SCT aims to improve social cognition, and ultimately social functioning through repeated practice with social stimuli (e.g., identifying emotions in pictures of faces), and compensatory strategy training (e.g., verbalization of salient facial features) [5]. A meta-analysis [6] of all types of SCT found large effects on emotion perception (recognition of emotions), and small to moderate effects on theory of mind (the ability to recognize and reflect on others' mental states). A more recent meta-analysis [7], including only comprehensive forms of SCT (i.e., targeting multiple areas of social cognition), found a large effect on emotion perception and theory of mind, and a small to moderate effect on attribution style (judgments about the causes of events and others' behavior). Together, these meta-analyses suggest that it is possible to improve social cognition with SCT in people with a psychotic disorder.

Some concerns have been raised, however, about the durability and generalizability of effects of SCT $[4,8]$. While one meta-analysis [6] found a positive effect of SCT on community functioning, other reviews point out that treatment effects are most commonly and robustly found for lower-order social cognition processes such as emotion perception. On higher-order social cognition such as theory of mind, and social functioning, findings are more mixed and dependent on the specific measure used $[4,8]$. We know from cognitive remediation research that integration with daily life is important for skills developed though cognitive training to generalize to social functioning [9]. Conventional SCT approaches may offer insufficient opportunity to practice in situations resembling real-life social interactions $[8,10]$. Thus, effects of SCT on higherorder social cognitive processes and social functioning may be limited, as training materials may not sufficiently capture the complex, interactive and dynamic nature of real-life social situations.

The utilization of virtual reality (VR) for SCT has been proposed as a possible way to address this issue $[4,10]$. VR is highly immersive, interactive and dynamic, and elicits psychological reactions similar to those in daily life [11]. Therefore, it is well-suited to simulate a variety of social situations and adequately reflect their complexity. At the same time, VR is controllable, which facilitates structured practice, and allows for situations to be scripted, repeated, varied, and personalized. Moreover, VR is a safe, accessible way to practice; participants know the virtual interactions can be stopped at any time, and that they can practice without negative repercussions and fear of embarrassment in their real social lives. Finally, VR is practical, as a wide range of situations can be generated quickly and practiced from one treatment setting, without traveling or accompanying participants in their daily environment.

VR is therefore a promising method to provide SCT. However, to our knowledge, no controlled studies of VR SCT for people with a psychotic disorder are available. Only one study on VR SCT has been published; a case report with two participants [12]. Improvements in social cognition and social functioning were reported after twenty sessions of a VR SCT. VR has also been used for social skills training (SST) in people with psychosis. A RCT of VR SST [13] among inpatients with schizophrenia $(n=91)$ showed that, compared to conventional SST, VR SST produced greater improvements in assertiveness and conversational skills, but no improvement was found in non-verbal skills. A pilot study of VR SST [14] 
reported improvement in social functioning, and VR measures revealed improvements in emotion perception, assertiveness, and time spent in conversations. Finally, pilot studies of VR SCT in people with autism spectrum disorder have reported improved emotion perception [15-17], attribution [16], theory of mind [15, 17], and (self-reported) social functioning [17]. Together, these studies suggest that VR SCT is promising.

To this end, we developed a novel VR SCT, Dynamic Interactive Social Cognition Training in Virtual Reality (DiSCoVR). In this randomized controlled trial (RCT), we aim to compare DiSCoVR to an active control condition, i.e., VR relaxation training (VRelax), in order to evaluate its efficacy on social cognition and social functioning. Moreover, we aim to assess the impact of the intervention on (the frequency and experience of) daily life social interactions, by using Experience Sampling Methodology (ESM), a diary method. We will also assess the durability of potential treatment effects in a 3-month follow-up period. Finally, we will perform moderator analysis to investigate who benefits from this VR SCT, and mediator analyses to examine variables mediating treatment effects. Thus, this study is the first RCT on VR SCT, using conventional measures of social cognition and functioning as well as experience sampling.

\section{Methods/design}

Participants

Participants are recruited from Dutch mental health treatment centers (University Center of Psychiatry of the University Medical Center Groningen, GGZ Drenthe, GGZ Delfland, and Zeeuwse Gronden). We recruit participants in two ways: 1) through advertisement of the study, by distributing posters and flyers, allowing participants to enroll themselves; and 2) through clinician referral: clinicians refer eligible and interested participants. Inclusion criteria are:

- A diagnosis of a psychotic disorder according to DSM-5 criteria, either determined by a structured clinical interview (e.g., Mini-international Neuropsychiatric Interview [18], Structured Clinical Interview for DSM [19], Schedules for Clinical Assessment in Neuropsychiatry [20]) in the past three years or verified using the MINI Plus at baseline.

- Deficits in social cognition, as indicated by a clinician

- Age 18-65 years.

Exclusion criteria are:

- (Photosensitive) Epilepsy.
- An estimated IQ below 70, and/or a diagnosis of intellectual disability.

- Insufficient proficiency in the Dutch language.

Participants receive a compensation of $€ 30$ for each completed assessment, up to a total of $€ 90$. They also receive reimbursement for travel expenses incurred for the assessments.

\section{Design}

The CONSORT inclusion flow diagram is shown in Fig. 1. This study is a multicenter single-blind randomized controlled trial (RCT) with two groups: 1) Dynamic Interactive Social Cognition Training in Virtual Reality (DiSCoVR), the experimental group; and 2) Virtual Reality Relaxation (VRelax), an active control group. Both groups receive treatment as usual alongside their designated intervention (DiSCoVR or VRelax). Participants complete three assessments: before the intervention (baseline, $\mathrm{T}_{0}$ ), directly after the intervention (post-treatment, $\mathrm{T}_{1}$ ) and three months after completing the intervention (follow-up, $\mathrm{T}_{2}$ ). After completion of the study, participants have the opportunity to receive the other treatment, if they wish.

\section{Interventions}

DiSCoVR

DiSCoVR is a 16-session individual VR SCT, taking place twice a week over a period of eight weeks. DiSCoVR is provided by psychologists with (at minimum) a master's degree in clinical psychology. A detailed treatment protocol describes the intervention, the software, the techniques that are used and the content of each session. Participants receive a workbook, containing session content summaries, exercises and worksheets (e.g., registration form of emotions they identified in their daily environment). DiSCoVR involves mass practice and compensatory strategy training. References to the patient's personal situations and goals are made in examples and/or exercises whenever possible, to facilitate generalization of training material to daily life. To the same end, through homework exercises, participants are encouraged to practice with techniques and strategies they have learned in the intervention in daily life. These homework exercises are discussed at the start of each session. The intervention consists of three parts, structured to build upon each other in increasing complexity:

- Module 1: Emotion perception (5 sessions, 1-5). Participants explore a virtual shopping street where they identify emotions on virtual characters' faces. Several characteristics (e.g., intensity of emotions, time to respond) can be altered to adjust the difficulty level to the training needs of the 


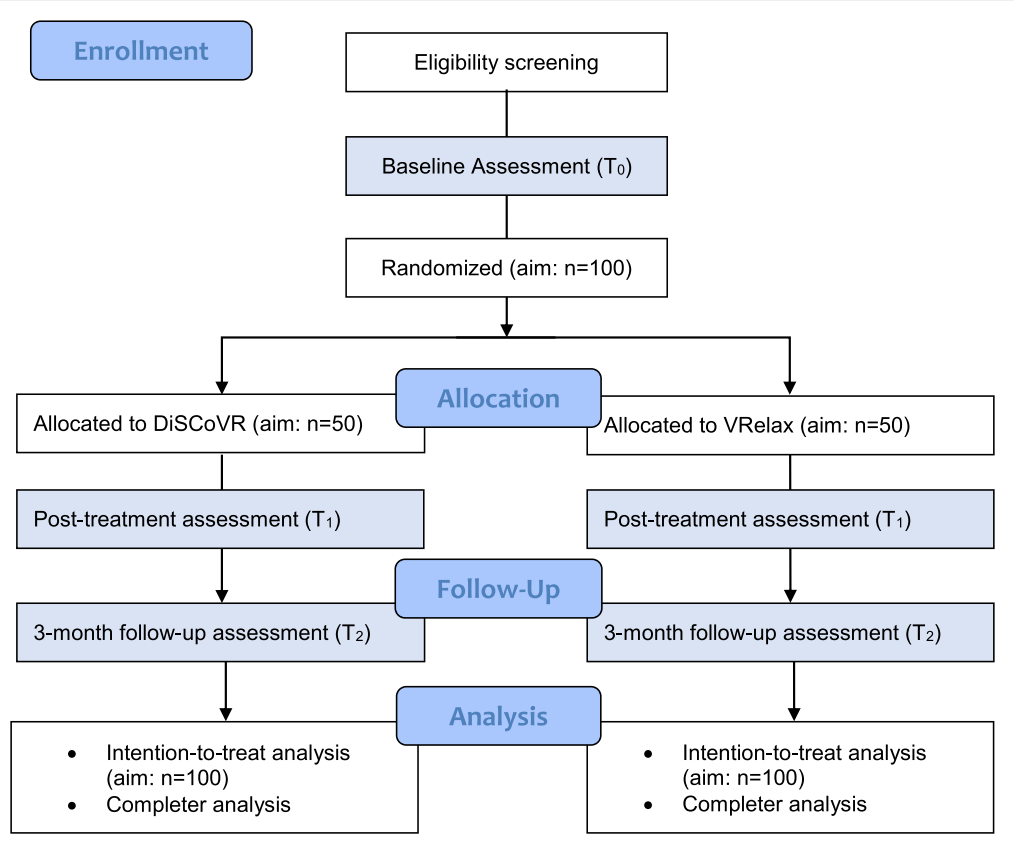

Fig. 1 CONSORT flow diagram

participant. Participants practice with several strategies (e.g., mimicking emotions) to aid in emotion recognition, which they are encouraged to try at home.

- Module 2: Social perception \& theory of mind (4 sessions, 6-9). Participants view scripted social scenarios in VR, about which open-ended questions regarding behavior, emotions and thoughts are asked. These questions aim to stimulate mentalization. Participants can respond by stating their answer out loud. If they answer incorrectly, characters will give more explicit hints about their mental state, with intensified emotional expression. Participants continue to apply social strategies to understand situations (e.g., remembering how you felt in a similar situation), by choosing a strategy before practicing in VR. Participants learn about the connection between thoughts, emotions and behavior, and practice with recognizing these in VR scenarios, themselves, and in others around them.

- Module 3: Social interaction (7 sessions, 10-16). Participants apply social cognitive skills in VR roleplay exercises with the therapist. They can practice with situations they struggle with, or that help them to achieve the goals they have set for the intervention. The protocol provides sample roleplay exercises, targeted at various social cognitive skills (e.g., detection of sarcasm and hints). Outside of VR, participants continue to practice with identifying emotions, thoughts and behavior and understanding their interrelatedness. They also learn a social problem-solving technique, in which they 1) consider their own thoughts, emotions and behavior; 2) consider the other person's thoughts, emotions and behavior; 3) consider different possible ways to react to a situation; and 4) role-play the different reactions and choose the most appropriate reaction to carry out in real life. Participants continue to practice using strategies (e.g., watching someone's emotional response to you to determine whether you should talk to them), both in VR and in real life.

The immersive VR worlds (a shopping street, a super market, an office and a bar), developed by CleVR BV, are displayed with an Oculus Rift headset (Consumer Version 1). They are shown in Fig. 2. Participants explore and interact with the virtual environment using a joystick controller. For the role play exercises, voice transformation is applied to mask the therapist's real voice. During the role plays, therapists can click buttons on the computer interface to trigger emotions and/or gesture animations (e.g., shrugging, pointing) in their virtual character.

\section{VRelax}

As an active control group, we chose VRelax, a VR relaxation therapy, as it matched important nonspecific characteristics of DiSCoVR (e.g., the use of VR, individual contact with a therapist twice a week), but did not target sociocognitive processes and therefore was not expected to improve social cognition. VRelax consists of sixteen sessions, provided twice a week in a period of 

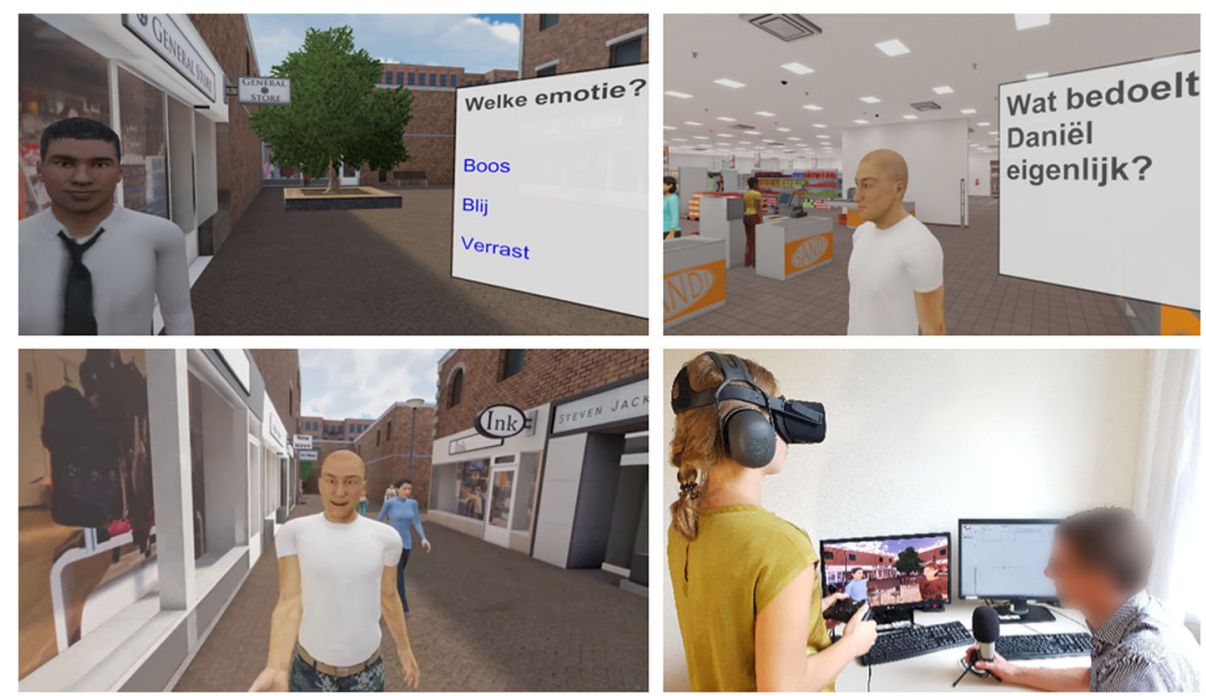

Fig. 2 Pictures of DiSCoVR environments and set-up. Legend: Top left: module 1 (translation: which emotion? Angry, happy, surprised); top right: module 2 (translation: what does Daniël actually mean?); bottom left: module 3, as seen from perspective of participant; bottom right: module 3, interactive role play setup. The virtual environments are property of CleVR BV; images have been used with permission from CleVR BV

eight weeks (the same as DiSCoVR), and aims to reduce both momentary (during sessions) and daily life stress and anxiety. VRelax is provided by therapists who either have 1) clinical experience in treating people with psychosis (e.g., psychiatric nurses); or 2) at least a bachelor's degree in psychology. In VRelax, participants' stressors are explored (i.e., which situations are stressful, and what are signs of stress). Participants learn to recognize stressful thoughts and rumination, and to apply coping strategies to address these (e.g., solving problems, asking for support and comfort, finding distraction). They also practice with relaxation exercises, such as breathing exercises, a body scan, grounding, and progressive muscle relaxation. Participants are encouraged to practice these techniques at home.

Each session starts with a discussion of stressors and negative emotions in the past few days. In some sessions, this is followed by psycho-education (e.g., about stress, coping and rumination). A large portion $( \pm 25 \mathrm{~min})$ of each session is reserved for relaxation in VR. Immersive $360^{\circ}$ video clips are shown with a Samsung Gear VR headset, running VRelax software developed by Viemr BV. Participants can visit a range of relaxing environments (Fig. 3), such as a sea in which they can swim with dolphins, a coral reef, a beach and a mountain meadow. They can control the environment using gaze; for example, to switch between environments or to engage with interactive elements (e.g., popping air bubbles underwater). Meditation exercises are embedded in the VR environment (e.g., guided meditation). Finally, part of each session is reserved for (learning to apply) relaxation techniques. These can be skipped if participants prefer to spend more time in VR.

\section{Materials and measurement instruments}

The measures used in this study are summarized in Table 1.

\section{Primary outcome}

Facial affect recognition Ekman 60 Faces, Facial Expression of Emotions Stimuli and Tests (FEEST) [21]. 60-item computerized picture test assessing facial affect recognition. Participants view pictures of six emotions (happy, sad, angry, disgusted, surprised, anxious) and identify the expressed emotion. Total scores (0-60) are used for analysis.

Social perception and theory of mind The Awareness of Social Inference Test part III (TASIT, Dutch version) $[22,23]$. Short video clips showing social situations containing lies and sarcasm. Participants answer four questions after each video about the intentions, intended messages, beliefs and emotions of the characters. Total scores (0-32) are used for analysis. Parallel versions are used; the administration order (A-B-A or B-A-B) is randomized across participants.

\section{Secondary outcomes}

Social functioning Personal and Social Performance Scale (PSP) [24]. An interview assessing the degree of disability (from 1, "absent", to 5, "severe") in four areas of social functioning: 1) socially meaningful activities (e.g., work, study); 2) personal and social relationships (e.g., friendships); 3) self-care (e.g., hygiene); and 4) 

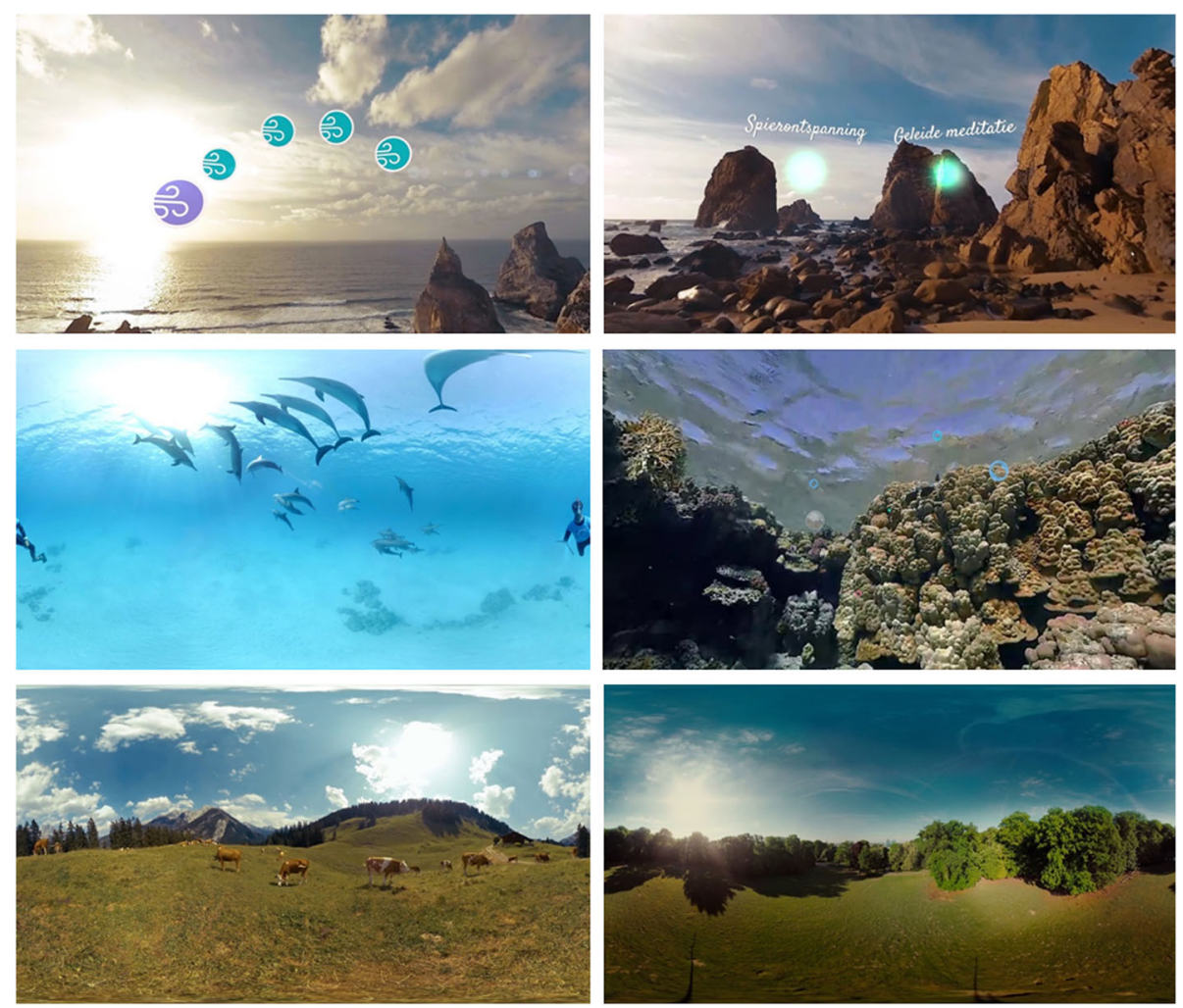

Fig. 3 VRelax environments, including relaxation exercises. The virtual environments are property of Viemr BV; images have been used with permission from Viemr BV

disturbing and aggressive behavior (e.g., problems with law enforcement). The ratings for each domain are combined into one score from 0 (very low) to 100 (very high), which will be used for analysis.

\section{Momentary mental states and social functioning in} daily life Experience Sampling Method (ESM) dairies are used to study the effects of the interventions on daily life interactions. An online questionnaire (21-33 items, included in Additional file 1: Table S1) is administered ten times a day for seven days at semi-random moments, by sending participants text messages containing a link to the questionnaire, which they can complete in the internet browser on a smartphone. Participants use their own smartphone or one provided by the research team. The ESM questionnaire assesses: 1) emotional state (positive and negative affect and stress level; thirteen items), on a visual analogue scale (VAS; 0-100); 2) current activities and activities since the last questionnaire (five items, to assess social and societal participation), selected from a multiple choice menu, and a VAS $(0-100)$ on the enjoyability of the activity; 3$)$ current social interactions and interactions since the last questionnaire (fourteen items), including the enjoyability of the interaction, feelings of being accepted, knowing how to respond, and feeling able to tell what the other person is feeling (VAS, 0-100); and 4) taking social initiative (two items; VAS, 0-100).

Psychiatric symptoms The following measures of psychopathology will be administered, since (social) cognitive training may improve psychiatric symptoms, and symptom levels could be a moderator or mediator of treatment effect:

- The Positive and Negative Syndrome Scale (PANSS) [25], a semi-structured interview assessing positive (7 items, range 7-49), negative (7 items, range 7-49) and general (16 items, range 16-112) symptoms of psychosis in the past week on a 7-point scale (from 1, "absent", to 7, "very severe"). We will analyze the three subscales separately as well as the total score.

- The Social Interaction Anxiety Scale (SIAS) [26], a 20-item self-report questionnaire measuring verbal and non-verbal social anxiety on a 5-point Likert scale. We will analyze the total score.

- The Green Paranoid Thought Scale (GPTS) [27], a self-report questionnaire measuring paranoid thoughts of social reference ([16] items, part A) and social persecution (16 items, part B) in the past 
Table 1 Measures used in this study

\begin{tabular}{|c|c|c|c|c|c|}
\hline Domain & Instrument & Method & Baseline $\left(T_{0}\right)$ & Post-treatment $\left(\mathrm{T}_{1}\right)$ & Follow-up $\left(T_{2}\right)$ \\
\hline \multicolumn{6}{|l|}{ Primary outcome measures } \\
\hline Emotion Perception & Ekman 60 Faces (FEEST) & Picture task & $x$ & $x$ & $x$ \\
\hline Social Perception / ToM & TASIT-III & Video task & $x$ & $x$ & $x$ \\
\hline \multicolumn{6}{|l|}{ Secondary outcome measures } \\
\hline Social functioning in daily life & $\begin{array}{l}\text { Questions about social interactions, } \\
\text { activities and momentary mental states }\end{array}$ & ESM questionnaire & $x$ & $x$ & $x$ \\
\hline Social functioning & PSP & Interview & $x$ & $x$ & $x$ \\
\hline Psychotic Symptoms & PANSS & $\begin{array}{l}\text { Semi-structured } \\
\text { interview }\end{array}$ & $x$ & $x$ & $x$ \\
\hline Social Anxiety & SIAS & Questionnaire & $x$ & $x$ & $x$ \\
\hline Paranoid Thoughts & GPTS & Questionnaire & $x$ & $x$ & $x$ \\
\hline Depression & BDI & Questionnaire & $x$ & $x$ & $x$ \\
\hline Anxiety & BAl & Questionnaire & $x$ & $x$ & $x$ \\
\hline Stress & PSS & Questionnaire & $x$ & $x$ & $x$ \\
\hline Self-esteem & SERS & Questionnaire & $x$ & $x$ & $x$ \\
\hline Information processing & TMT & Task & $x$ & $x$ & $x$ \\
\hline $\begin{array}{l}\text { Demographic and clinical } \\
\text { background }\end{array}$ & Questions & Interview & $x$ & $x$ & $x$ \\
\hline \multicolumn{6}{|l|}{ Baseline diagnostic measures } \\
\hline Premorbid intelligence & NLV & Task & $x$ & & \\
\hline Diagnostic & M.I.N.I. Plus & Structured Interview & $x$ & & \\
\hline \multicolumn{3}{|l|}{ Measures during sessions } & \multicolumn{3}{|l|}{ Session(s) } \\
\hline Cyber sickness & SSQ & Questionnaire & S3 & & \\
\hline Emotions & Questions & Questionnaire & VRelax: each & session $(2 x)$ & \\
\hline Performance on VR task & Social cognition tasks in VR & Task & DiSCoVR: S1- & & \\
\hline Behavior in VR & Reaction time, interpersonal distance & Task & DiSCoVR: S1- & & \\
\hline
\end{tabular}

month on a 5-point Likert scale. We will analyze both subscales separately.

- Perceived Stress Scale (PSS) [28], a ten-item selfreport questionnaire on symptoms of stress in the past week, indicated on a 5-point Likert scale. We will analyze the total score.

\section{Other}

Self-esteem The Self-Esteem Rating Scale (SERS) [29]. The SERS is a twenty-item self-report questionnaire using a 7-point Likert scale, assessing feelings of selfworth and competence. Total scores will be analyzed.

Information processing and executive functioning The Trailmaking Test (TMT) [30]. In TMT-A, participants connect numbers in circles scattered across a page in consecutive order; in TMT-B, participants connect numbers and letters in circles scattered across a page, alternating between the letters and circles in consecutive order (1-A-2-B, etc.). The completion time in seconds for both parts is analyzed.

Demographic and clinical background Questions on different clinical and demographic variables, including: age, gender, handedness, eyesight, family history of psychiatric illness, education level of participant and participant's parents, work history, diagnosis and illness duration (administered only at baseline); and housing status, employment status, substance use, medication use, hospitalizations and psychotic episodes (administered at each assessment).

Premorbid intelligence The Dutch version (Nederlandse Leestest voor Volwassenen, NLV) [31] of the National Adult Reading Test [32]. Fifty increasingly uncommon words are read aloud by participants. Correct pronunciations are awarded a score of two points. 
The total score is used for analysis. The NLV is only administered at baseline.

Eligibility check Mini International Neuropsychiatric Interview Plus (M.I.N.I.-plus) [18]. A structured clinical interview on symptoms of psychosis, depression and mania. The MINI-plus is administered at baseline to verify a diagnosis of psychotic disorder, if a diagnosis has not been determined by a structured clinical interview in the past three years.

\section{Measures during treatment sessions}

Cyber sickness Simulator Sickness Questionnaire (SSQ) [33]. A self-report questionnaire on 27 common symptoms of simulator sickness (e.g., headache, nausea, dizziness), which will be administered right after the VR session 3 of both interventions to evaluate negative side effects of using VR. We will use the total score for analysis.

Performance and behavior during VR tasks In the DiSCoVR group, the VR program will log several parameters during practice sessions (e.g., the avatars encountered, answers given to questions, time taken to answer, the number of answer attempts and interpersonal distance).

Emotions before and after relaxation In the VRelax group, before and after each session in VR, participants complete a short questionnaire in which they rate their emotions (relaxed, anxious, gloomy, tranquil, stressed, excited, nervous and content) on a 0-100 VAS.

\section{Assessment}

The assessments are carried out by trained, independent research assistants. This study is single-blind, meaning that research assistants do not know to which intervention participants have been assigned. This is achieved by instructing coordinators, therapists and participants not to divulge group allocation to research assistants. Other precautions include storing data revealing group allocation (e.g., therapist worksheets) in a separate location and using different assistants for each measurement as much as possible. Blinding is evaluated with a self-report form for research assistants at the end of the post-treatment and follow-up assessments. We will perform a sensitivity analysis by testing the treatment effect only for measurements where research assistants reported being completely blinded to group allocation.

\section{Randomization procedure}

We utilized permuted block randomization with randomized block sizes (2, 4 or 6$)$ using the $\mathrm{R}$ package 'blockrand' [34]. For each participating treatment center, we created strata for age (ten-year increments) and gender (i.e., ten strata per center). This resulted in a master randomization list with either 'A' or 'B' in each stratum. An independent employee then added an additional randomization by assigning DiSCoVR or VRelax to either $\mathrm{A}$ or $\mathrm{B}$ in the master randomization list using coin flips, once for each treatment center. Upon completing $\mathrm{T}_{0}$, the participants will be placed in the appropriate stratum in order of inclusion by the independent randomizer, determining their group assignment.

\section{Protocol fidelity}

Therapists in both conditions will complete an eighthour training. Monthly group supervision, in which all ongoing cases are discussed, will be provided for both conditions, to aid therapists and improve protocol fidelity. Therapists who are unavailable for these meetings will receive individual supervision. A weekly consultation session with the research team will also be available. After each session, therapists will complete a session form, on which they indicate whether they completed all parts of the protocol for that session, and whether any anomalies occurred.

\section{Drop-out}

Participants will be able to discontinue the study at any point. They can also refuse participation in part of the study, such as the ESM questionnaires. For sensitivity analyses, participants will be considered treatment completers if they complete twelve sessions or more (> 75\%). If participants drop out of treatment, they are asked to continue the assessments. If participants refuse or miss $\mathrm{T}_{1}$, they are still contacted for $\mathrm{T}_{2}$.

\section{Data management}

Participant data will be coded using a study ID. Personal information and informed consent will be stored separately to ensure anonymity. We will use an electronic case report form; paper source data will be stored securely at each respective location. An independent monitor, who performs yearly routine inspections, will be appointed to ensure study quality and integrity.

\section{Analysis}

For our primary outcome variables (theory of mind and emotion perception), previous meta-analyses $[6,7]$ have found effect sizes ranging between moderate $(d=.46$, theory of mind) and large ( $d=.84$, emotion perception). We therefore assumed a conservative effect size of .5. With $\beta=.80, \alpha=.05$, two groups and three assessments, we found we would need 86 participants. Assuming a dropout rate of $13 \%$ [35], we concluded that the sample should consist of 100 participants (50 in each group). 
The groups will be compared at baseline using t-tests for normally distributed variables, and Mann-Whitney U-test or $\chi^{2}$ test for other variables. Variables with a significant baseline difference between the groups will be added to the analysis as a covariate.

We will use multilevel mixed-model linear regression analysis in accordance with the intention to treat principle, with repeated assessments (level 1) nested within participants (level 2). Time and group and their interaction term will be included as fixed effects. Treatment effects will be evaluated by examining the interaction between time and group. A random intercept will be included for the participant level to account for the repeated measures. A random slope will be included for time if this improves the model, according to the AIC value. The post-treatment and follow-up assessments will be analyzed separately, comparing each to the baseline. We will also perform a multilevel regression analysis on treatment completers, defined as people who have completed at least twelve sessions (75\%) of their allocated intervention. Furthermore, moderator (e.g., clinical and demographic variables) and mediator analyses will also be conducted. Significance will be accepted at $\alpha=.05$ (two-tailed); in case of multiple measurements within the same domain, a correction will be applied to $\alpha$.

\section{Procedure}

Clinicians will approach potential participants and gauge their interest in the study. If patients express interest, or if potential participants approach the research team directly, they will be contacted and screened by the research team. They will receive written information about the study and will be given a one-week consideration period. If they are still interested after this period, participants will provide written informed consent, collected by the research assistant, and the baseline assessment will take place. In the week after the first assessment, the baseline ESM questionnaire period will take place. After this, participants are randomized to either DiSCoVR or VRelax, and receive sixteen DiSCoVR or VRelax sessions. When treatment has been completed, the post-treatment assessment will take place, followed by another ESM period of one week. Finally, three months after completing treatment, participants will complete the last follow-up assessment, followed by the final ESM questionnaire week.

\section{Discussion}

The main goal of this trial is to evaluate the effect of DiSCoVR on social cognition and social functioning. We hypothesize that the DiSCoVR group will show greater improvements in emotion perception and theory of mind than VRelax at post-treatment, and that this difference will be maintained at 3-month follow-up. Additionally, we expect that participants in the DiSCoVR group will experience greater improvement in social functioning, as measured by an interview (PSP). Furthermore, we hypothesize that people in the DiSCoVR group will show more improvement in daily-life social functioning (i.e., feelings of acceptance, subjective social cognition, social participation and social initiative) than people who receive VRelax. Since VRelax targets stress, anxiety, coping and stress reactivity, we expect that participants in the control group will show equal or larger improvements in perceived stress and general anxiety.

Very little is currently known about mediators and moderators of treatment effects of SCT [4]. Therefore, mediation and moderation analyses will be explorative in nature. A meta-analysis on SCT [6] found that illness duration and education level were moderators of treatment effects on social cognition. We therefore postulate that these variables may moderate the treatment outcome of DiSCoVR, but we will also investigate other moderators (e.g., baseline levels of social anxiety and paranoia).

However, no meta-analysis thus far has investigated mediators of SCT efficacy. Both Eack et al. [36] and Hogarty et al. [37] found that improvements in neurocognition mediated improvements in social functioning after Cognitive Enhancement Training, a form of SCT targeting both social cognition and neurocognition. Neurocognition may therefore be a mediator of treatment outcome in the present study. However, since little is currently known about mediators of SCT treatment effects, we will explore additional variables.

While SCT is not a new method, the present study intends to improve upon existing methods by allowing participants to apply social cognition in personalized virtual environments and situations resembling daily life. This trial will also address some of the gaps in knowledge about SCT [4], particularly concerning generalization to daily life and durability, but also moderators and mediators of training effects.

Since there are presently no RCTs on VR SCT, the present study will shed light on the efficacy of this innovative method. If it proves to be effective, it could be an important tool to improve social cognition and social functioning which is sorely needed, since durable functional recovery after psychosis is still uncommon [38]. Finally, because social cognitive deficits are a transdiagnostic characteristic [39], VR SCT may be a useful tool to remediate problems in social cognition and social functioning across a range of psychiatric populations, such as autism spectrum disorders.

\section{Additional file}

Additional file 1: Table S1. ESM questionnaire. (DOCX $21 \mathrm{~kb}$ ) 
ESM: Experience Sampling Method(ology); FEEST: Facial Expressions of Emotion: Stimuli and Tests; GPTS: Green Paranoid Thought Scale; M.I.N.I.plus: Mini International Neuropsychiatric Interview Plus; NART: National Adult Reading Test; NLV: Nederlandse Leestest voor Volwassenen; PANSS: Positive and Negative Syndrome Scale; PSP: Personal and Social Performance Scale; PSS: Perceived Stress Scale; RCT: Randomized Controlled Trail; S: Session; SCT: Social Cognition Training; SERS: Self-Esteem Rating Scale; SIAS: Social Interaction Anxiety Scale; SSQ: Simulator Sickness Questionnaire; $T_{0}$ : Baseline; $\mathrm{T}_{1}$ : Post-treatment; $T_{2}$ : Follow-up; TASIT: The Awareness of Social Inference Test; TMT: Trailmaking Test; UMCG: University Medical Center Groningen; VR: Virtual Reality; VRelax: Virtual Reality Relaxation

\section{Acknowledgements}

We thank all participants and therapists who took part in our pilot study of DiSCoVR, for their valuable feedback towards improving the intervention and protocol. We also thank the four UMCG patients who provided us with feedback on our RCT study materials. Finally, we thank all site coordinators, therapists, participants, research assistants and referring clinicians who make this study possible.

\section{Authors' contributions}

GP, WW, SN, CG and KGL conceived and designed the study. SN, GP, WV and KGL wrote the DiSCoVR protocol, with contributions from MV and CZ. SN, GP, WV and CG conceived the concept and content of the VR program and supervised its development. RV, CZ and DZ arranged for and coordinated study participation at their respective locations. SN wrote the first draft of the manuscript, which was critically revised by WV and GP. All authors have read, provided input to, and approved the final manuscript.

\section{Funding}

The development of the VR software was supported by a peer-reviewed $€ 75,000$ Knowledge Innovation Mapping grant (628.005.007), awarded by the Netherlands Organization for Scientific Research (NWO). GGZ Drenthe provided $€ 12,173$ for four VR headsets and supporting research staff through an internal research grant. Finally, the University of Groningen provided a staff grant of $€ 9,864$ for participant compensation and reimbursement of their travel expenses. None of these institutions have played a role in the design of the study, data collection, analysis, and interpretation of data or in writing the manuscript.

\section{Availability of data and materials}

The datasets used and/or analyzed during the current study will be available from the corresponding author on reasonable request.

\section{Ethics approval and consent to participate}

Written informed consent will be collected from all participants. This study is carried out in accordance with the Declaration of Helsinki and has been approved by the Medical Ethical Committee of the University Medical Center Groningen (METc file number: 2017/573, ABR: NL63206.042.17, protocol version 2, 07-12-2017). The study was also approved by the Committee for Research and Health Care Innovation (Commissie Onderzoek en Zorginnovatie, GGZ Drenthe), Committee for Scientific Research (Commissie Wetenschappelijk Onderzoek, GGZ Delfland), and the board of directors of Zeeuwse Gronden. (Serious) adverse events are recorded and reported to the independent monitor and the Medical Ethical Committee.

\section{Consent for publication}

Not applicable.

\section{Competing interests}

The authors declare that they have no competing interests.

\section{Author details}

1Department of Psychotic Disorders, GGZ Drenthe, Dennenweg 9, PO Box 30007, 9404, LA, Assen, the Netherlands. ${ }^{2}$ University Center of Psychiatry, University Medical Center Groningen, University of Groningen, Hanzeplein 1, PO Box 30.001, 9700, RB, Groningen, the Netherlands. ${ }^{3}$ Department of Child and Adolescent Psychiatry/Psychology, Erasmus MC-Sophia, Wytemaweg 8, 3015, CN, Rotterdam, The Netherlands. ${ }^{4}$ Autism Team Northern-Netherlands of Jonx, Department of (Youth) Mental Health and Autism of Lentis Psychiatric Institute, Laan Corpus den Hoorn 102-2, 9728, JR, Groningen, The
Netherlands. ${ }^{5}$ Department of Yulius Autism, Yulius, Amazone 7, 3315, WG, Dordrecht, the Netherlands. ${ }^{6}$ Flexible Assertive Community Treatment Team, Outpatient Treatment Center, GGZ Delfland, Sint Jorisweg 2, 2612, GA, Delft, The Netherlands. 'Zeeuwse Gronden, Axelsestraat 8/A, 4537, AJ, Terneuzen, The Netherlands. ${ }^{8}$ Department of Psychology, University of Groningen, Grote Kruisstraat 2/1, 9712, TS, Groningen, The Netherlands.

\section{Received: 8 May 2019 Accepted: 20 August 2019}

Published online: 05 September 2019

\section{References}

1. Couture SM, Penn DL, Roberts DL. The Functional Significance of Social Cognition in Schizophrenia: A Review. Schizophr Bull. 2006;32 Supplement 1:S44-63. doi:https://doi.org/10.1093/schbul/sbl029.

2. Savla GN, Vella L, Armstrong CC, Penn DL, Twamley EW. Deficits in domains of social cognition in schizophrenia: a meta-analysis of the empirical evidence. Schizophr Bull. 2013;39:979-92. https://doi.org/10.1093/schbul/ sbs080.

3. Fett A-K, Viechtbauer W, Penn DL, van Os J, Krabbendam L. The relationship between neurocognition and social cognition with functional outcomes in schizophrenia: a meta-analysis. Neurosci Biobehav Rev. 2011;35: $573-88$.

4. Horan WP, Green MF. Treatment of social cognition in schizophrenia: current status and future directions. Schizophr Res. 2017. https://doi.org/10.1 016/j.schres.2017.07.013.

5. Paquin K, Wilson AL, Cellard C, Lecomte T, Potvin S. A systematic review on improving cognition in schizophrenia: which is the more commonly used type of training, practice or strategy learning? BMC Psychiatry. 2014;14:139.

6. Kurtz MM, Richardson CL. Social cognitive training for schizophrenia: a meta-analytic investigation of controlled research. Schizophr Bull. 2012;38: 1092-104.

7. Kurtz MM, Gagen E, Rocha NBF, Machado S, Penn DL. Comprehensive treatments for social cognitive deficits in schizophrenia: a critical review and effect-size analysis of controlled studies. Clin Psychol Rev. 2016;43:80-9.

8. Fiszdon JM, Reddy LF. Review of social cognitive treatments for psychosis. Clin Psychol Rev. 2012;32:724-40.

9. Medalia A, Saperstein AM. Does cognitive remediation for schizophrenia improve functional outcomes? Curr Opin Psychiatry. 2013;26:151-7.

10. Peyroux E, Franck N. RC2S: a cognitive remediation program to improve social cognition in schizophrenia and related disorders. Front Hum Neurosci. 2014;8. https://doi.org/10.3389/fnhum.2014.00400.

11. Veling W, Moritz S, van der Gaag M. Brave new worlds - review and update on virtual reality assessment and treatment in psychosis. Schizophr Bull. 2014:40:1194-7. https://doi.org/10.1093/schbul/sbu125.

12. Peyroux E, Franck N. Improving social cognition in people with schizophrenia with RC2S: two single-case studies. Front Psychiatry. 2016;7. https://doi.org/10.3389/fpsyt.2016.00066.

13. Park K-M, Ku J, Choi S-H, Jang H-J, Park J-Y, Kim SI, et al. A virtual reality application in role-plays of social skills training for schizophrenia: a randomized, controlled trial. Psychiatry Res. 2011;189:166-72. https://doi. org/10.1016/j.psychres.2011.04.003.

14. Rus-Calafell M, Gutiérrez-Maldonado J, Ribas-Sabaté J. A virtual realityintegrated program for improving social skills in patients with schizophrenia: a pilot study. J Behav Ther Exp Psychiatry. 2014;45:81-9. https://doi.org/10.1016/j.jbtep.2013.09.002.

15. Yang YJD, Allen T, Abdullahi SM, Pelphrey KA, Volkmar FR, Chapman SB. Brain responses to biological motion predict treatment outcome in young adults with autism receiving virtual reality social cognition training: preliminary findings. Behav Res Ther. 2017;93:55-66. https://doi.org/10.1016/ j.brat.2017.03.014.

16. Didehbani N, Allen T, Kandalaft M, Krawczyk D, Chapman S. Virtual reality social cognition training for children with high functioning autism. Comput Hum Behav. 2016;62:703-11. https://doi.org/10.1016/..chb.2016.04.033.

17. Kandalaft MR, Didehbani N, Krawczyk DC, Allen T, Chapman SB. Virtual reality social cognition training for young adults with high-functioning autism. J Autism Dev Disord. 2013;43:34-44. https://doi.org/10.1007/s10803012-1544-6 Accessed 26 May 2015.

18. Sheehan DV, Lecrubier Y, Sheehan KH, Amorim P, Janavs J, Weiller E, et al. The Mini-International Neuropsychiatric Interview (M.I.N.I.): The Development and Validation of a Structured Diagnostic Psychiatric Interview for DSM-IV and ICD-10. J Clin Psychiatry. 1998;59 suppl 20:22-33. 
19. First MB. Structured clinical interview for the DSM (SCID). Encycl Clin Psychol. 2014:1-6.

20. Wing JK, Sartorius N, Üstün TB. Diagnosis and clinical measurement in psychiatry: a reference manual for SCAN: Cambridge University Press; 1998.

21. Young A, Perrett D, Calder A, Sprengelmeyer R, Ekman P. Facial expressions of emotion: stimuli and tests (FEEST). Thames Valley Test Company: Bury St Edmunds, UK; 2002.

22. McDonald S, Flanagan S, Rollins J, Kinch J. TASIT: a new clinical tool for assessing social perception after traumatic brain injury. J Head Trauma Rehabil. 2003;18:219.

23. Westerhof-Evers HJ, Visser-Keizer AC, McDonald S, Spikman JM. Performance of healthy subjects on an ecologically valid test for social cognition: the short, Dutch version of the awareness of social inference test (TASIT). J Clin Exp Neuropsychol. 2014;36:1031-41. https://doi.org/10.1080/13803395.2014.966661.

24. Kawata AK, Revicki DA. Psychometric properties of the personal and social performance scale (PSP) among individuals with schizophrenia living in the community. Qual Life Res Int J Qual Life Asp Treat Care Rehabil. 2008;17: 1247-56.

25. Kay SR, Fiszbein A, Opler LA. The positive and negative syndrome scale (PANSS) for schizophrenia. Schizophr Bull. 1987;13:261-76. https://doi.org/1 0.1093/schbul/13.2.261.

26. Osman A, Gutierrez PM, Barrios FX, Kopper BA, Chiros CE. The social phobia and social interaction anxiety scales: evaluation of psychometric properties. J Psychopathol Behav Assess. 1998;20:249-64. https://doi.org/10.1023/A:1023 067302227.

27. Green CEL, Freeman D, Kuipers E, Bebbington P, Fowler D, Dunn G, et al. Measuring ideas of persecution and social reference: the Green et al. paranoid thought scales (GPTS). Psychol Med. 2008;38:101-11.

28. Cohen S, Kamarck T, Mermelstein R. A global measure of perceived stress. J Health Soc Behav. 1983;24:385

29. Lecomte T, Corbière M, Laisné F. Investigating self-esteem in individuals with schizophrenia: relevance of the self-esteem rating scale-short form. Psychiatry Res. 2006;143:99-108.

30. Reitan RM. Trail making test: manual for administration and scoring. Reitan Neuropsychology Laboratory: Tuscon, Arizona, United States; 1992.

31. Lindeboom J, Schmand B, van Harskamp F. NLV. Nederlandse Leestest voor Volwassenen. Handleiding. Lisse, NL: Swets \& Zeitlinger; 1992.

32. Nelson HE, Willison J. The National Adult Reading Test (NART). Nfer-Nelson: Windsor; 1991

33. Kennedy RS, Lane NE, Berbaum KS, Lilienthal MG. Simulator sickness questionnaire: an enhanced method for quantifying simulator sickness. Int J Aviat Psychol. 1993;3:203-20. https://doi.org/10.1207/s15327108ijap0303_3.

34. Snow G. blockrand: Randomization for Block Random Clinical Trials. 2018. https://cran.r-project.org/web/packages/blockrand/index.html.

35. Villeneuve $\mathrm{K}$, Potvin $\mathrm{S}$, Lesage A, Nicole L. Meta-analysis of rates of drop-out from psychosocial treatment among persons with schizophrenia spectrum disorder. Schizophr Res. 2010;121:266-70.

36. Eack SM, Pogue-Geile MF, Greenwald DP, Hogarty SS, Keshavan MS. Mechanisms of functional improvement in a 2-year trial of cognitive enhancement therapy for early schizophrenia. Psychol Med. 2011;41:125361. https://doi.org/10.1017/S0033291710001765.

37. Hogarty GE, Greenwald DP, Eack SM. Durability and mechanism of effects of cognitive enhancement therapy. Psychiatr Serv. 2006;57:1751-1757. doi: https://doi.org/10.1176/appi.ps.57.12.1751.

38. Jääskeläinen $\mathrm{E}$, Juola $\mathrm{P}$, Hirvonen $\mathrm{N}, \mathrm{McGrath} \mathrm{JJ}$, Saha S, Isohanni M, et al. A systematic review and meta-analysis of recovery in schizophrenia. Schizophr Bull. 2013;39:1296-306.

39. Kennedy DP, Adolphs R. The social brain in psychiatric and neurological disorders. Trends Cogn Sci. 2012;16:559-72. https://doi.org/10.1016/j.tics.2 012.09.006.

\section{Publisher's Note}

Springer Nature remains neutral with regard to jurisdictional claims in published maps and institutional affiliations.

Ready to submit your research? Choose BMC and benefit from:

- fast, convenient online submission

- thorough peer review by experienced researchers in your field

- rapid publication on acceptance

- support for research data, including large and complex data types

- gold Open Access which fosters wider collaboration and increased citations

- maximum visibility for your research: over $100 \mathrm{M}$ website views per year

At BMC, research is always in progress.

Learn more biomedcentral.com/submissions 